\title{
NEW APPROACH FOR BANDWIDTH SELECTION IN THE KERNEL DENSITY ESTIMATION BASED ON $\beta$-DIVERGENCE
}

\section{HAMZA DHAKER ${ }^{1}$, PAPA NGOM ${ }^{1}$, EL HADJI DEME ${ }^{2}$ and MALICK MBODJ ${ }^{3}$}

1LMA

Universit Cheikh Anta Diop de Dakar

Senegal

e-mail: hamzaould.yahya@ucad.edu.sn

2LERSTAD

Universite Gaston Berger

UFR SAT

Senegal

${ }^{3}$ Bowie State University

Maryland

USA

\begin{abstract}
The choice of bandwidth is crucial to the kernel density estimation (KDE). Various bandwidth selection methods for KDE, least squares cross-validation (LSCV) and Kullback-Leibler cross-validation are proposed. We propose a method to select the optimal bandwidth for the KDE. The idea behind this method is to generalize the LSCV method, using the measure of $\beta$-divergence;
\end{abstract}

2010 Mathematics Subject Classification: 94A17, $62 \mathrm{G} 07$.

Keywords and phrases: nonparametric density estimation, measure divergence, integrated squared error, bandwidth.

Received April 25, 2018; Revised May 14, 2018

(C) 2018 Scientific Advances Publishers 
and to see the improvement in our method, we will compare these $\mathcal{D}_{\beta}\left(\widehat{f}_{h}, f\right)$ bandwidth selector with a normal reference (NR), the last squares crossvalidation (LSCV), the Sheather and Jones (SJ) method, and the generalized $\operatorname{LSCV}\left(\operatorname{LSCV}_{g}\right)$ bandwidth selector, on simulated data. The use of the various practical bandwidth selectors is illustrated on a real data example.

\section{Introduction}

The problem of choosing the bandwidth (window width or smoothing parameter) $h$ is important in statistical estimation of the kernel density estimation. A vast amount of literatures has been devoted in choosing practical optimal bandwidth for techniques build on kernel estimation. Some comparative studies have been made on these methods.

Representative surveys of bandwidth selection techniques can be found in [3, 9, 12, 5, 24], and [18].

Least-squares cross-validation (LSCV) is among the earliest bandwidth selectors, this method was suggested by Rudemo [17] and Bowman [2], in the 80s, it has been the method of reference, but in the early 90 s, studies have shown that other methods performs better from the bias points of view and much better in reducing the variance. See Park and Turlach [15] for a detailed description.

Interesting comparative studies have been published. Bowman [2] compared two methods for selecting bandwidth: The first is the KullbackLeibler cross-validation and the second is that of integrated squared error cross-validation. Scott and Terrell [19] compared the two methods by theoretical calculation of the noise in the cross-validation function and corresponding cross-validated smoothing parameters, by Monte Carlo simulation, and by example.

Sheather and Jones [20] set up a plug-in type of three-step procedure. They choose to estimate $R\left(f^{\prime \prime}\right)$ (the term unknown in AMISE).

Zhang ([26]) have proposed a generalization the classical least squares cross-validation (LSCV) selector for its variability and undersmoothing, he did a comparison of bandwidths for finite sample behaviour. 
For a more complete treatment, from a historical viewpoint, with complete references, and detailed discussion of variations that have been suggested, see Jones et al. [10]. Quick access to implementation of most of the methods discussed here has been provided by Jones et al. [9].

The main purpose of this paper is to investigate the optimal bandwidth $h_{\beta}$ for minimizing criterion $\operatorname{ED}_{\beta}\left(\widehat{f}_{n}, f\right)$ error. We will see that $\operatorname{ED}_{\beta}\left(\widehat{f}_{n}, f\right)$ generalize the integrated square error (ISE) and Kullback-Leibler divergence (KL). After having introduced the $\mathcal{D}_{\beta}\left(\widehat{f}_{n}, f\right)$ selection method, we study the finite sample performances of various bandwidth selectors via a simulation study. We compare five procedures: the normal reference (NR) method, the least squares crossvalidation (LSCV), the Sheather and Jones (SJ) method, the generalized $\operatorname{LSCV}\left(\operatorname{LSCV}_{g}\right)$, and criterion $\mathcal{D}_{\beta}\left(\widehat{f}_{n}, f\right)$ error.

This paper is organized as follows. Section 2 describes the classical methods for bandwidth selections. Section 3 presents the new method proposed for bandwidth selector, which generalizes and provides improvement for the least squares cross-validation (LSCV). In Section 4, we will present some simulation results for estimation and do a comparison of the various methods. Section 5 applies the methods to real data. Finally, the conclusion and perspective will be presented in Section 6 .

\section{Classical Methods for Bandwidth Selection}

Given an $n$-sample $X_{1}, X_{2}, \ldots, X_{n}$ of independent random variables with an unknown density $f$, let us consider the Parzen-Rosenblatt kernel estimator of the density $f$ given by:

$$
\widehat{f}_{h}(x)=\frac{1}{n h} \sum_{i=1}^{n} \mathcal{K}\left(\frac{x-X_{i}}{h}\right),
$$

where $h>0$ is the bandwidth and $\mathcal{K}$ is a density function defined on $R$ called kernel. 
To estimate $f$, choose the kernel $\mathcal{K}$ and $h$ parameter. If the choice of the kernel is not a problem, it is not the case for the choice of the width of the window $h$ which essentially depends on the size $n$ of the sample. There are two methods of families: the family of cross-validation methods and the family of plug-in methods.

The decision of an optimal choice for the bandwidth necessitates the specification of an error criterion that can be optimized. By minimizing the mean integrated square error (MISE). In this case, [16] there is obtained

$$
\begin{aligned}
\operatorname{MISE}\left(\widehat{f}_{h}(x)\right) & =\int E\left[\widehat{f}_{h}(x)-f(x)\right]^{2} d x \\
& =\frac{h^{4}}{4} \mu_{2}(\mathcal{K})^{2} \int\left(f^{\prime \prime}(x)\right)^{2} d x+\frac{R(\mathcal{K})}{n h}+O\left(h^{5}+\frac{1}{n}\right),
\end{aligned}
$$

where $\mu_{2}(\mathcal{K})=\int x^{2} \mathcal{K}(x) d x$ is the variance of kernel $\mathcal{K}$ and $R(g)=\int g^{2}(x) d x$ for any function $g$.

The asymptotics mean integrated square error (AMISE) is then of the form:

$$
\operatorname{AMISE}\left(\widehat{f}_{h}\right)=\frac{h^{4}}{4} \mu_{2}(\mathcal{K})^{2} R\left(f^{\prime \prime}\right)+\frac{R(\mathcal{K})}{n h}
$$

To find the closed form expression for $h_{A M I S E}$, begin by differentiating (3) to obtain

$$
\frac{\partial A M I S E}{\partial h}=-\left(n h^{2}\right)^{-1} R(\mathcal{K})+h^{3} \mu_{2}(\mathcal{K})^{2} R\left(f^{\prime \prime}\right) .
$$

Setting this equation equal to 0 and solving for $h$ produces

$$
h_{\text {AMISE }}=\left[\frac{\mathcal{R}(\mathcal{K})}{n \mu_{2}(\mathcal{K})^{2} R\left(f^{\prime \prime}\right)}\right]^{1 / 5} .
$$

It is found that the optimal width of $h_{A M I S E}$ window depends on the unknown density $f$ through the parameter $R\left(f^{\prime \prime}\right)$, which has to be estimated before using $h_{A M I S E}$. 
A very natural way to get around the problem of not knowing $f^{\prime \prime}$ is to use a standard family of distributions to assign a value of the term $R\left(f^{\prime \prime}\right)$ in expression (4). For example, assume that a density $f$ belongs to the Gaussian family with mean $\mu$ and variance $\sigma$, then

$$
\begin{aligned}
R\left(f^{\prime \prime}\right)=\int\left(f^{\prime \prime}(x)\right)^{2} d x & =\sigma^{-5} \int\left(\phi^{\prime \prime}(x)\right)^{2} d x \\
& =\frac{3}{8} \pi^{-1} 2 \sigma^{-5} \approx 0.212 \sigma^{-5},
\end{aligned}
$$

where $\phi(x)$ is the standard normal density. If one uses a Gaussian kernel, then

$$
\begin{aligned}
h_{N R} & =(4 \pi)^{-1 / 10}\left(\frac{3}{8} \pi^{-1 / 2}\right)^{-1 / 5} \sigma n^{-1 / 5} \\
& =\left(\frac{4}{3}\right)^{1 / 5} \sigma n^{-1 / 5} .
\end{aligned}
$$

If we want to make this estimate more insensitive to outliers, we have to use a more robust estimate for the scale parameter of the distribution. Let $\widehat{R}$ be the sample interquartile, then one possible choice for $h$ is

$$
\begin{aligned}
h_{N R} & =1.06 \min \left(\widehat{\sigma}, \frac{\widehat{R}}{(\Phi(3 / 4)-\Phi(1 / 4))}\right) n^{-1 / 5} \\
& =1.06 \min \left(\widehat{\sigma}, \frac{\widehat{R}}{1.349}\right) n^{-1 / 5},
\end{aligned}
$$

where $\Phi$ is the standard normal distribution function. To see more detail, e.g., [21, 8, 18].

The LSCV, sometimes called an unbiased cross-validation was proposed by [17] and [2]. The criterion is to choose the bandwidth that minimizes an estimator of integrated square error (ISE):

$$
I S E=\int \widehat{f}_{h}^{2}(x) d x-2 \int \widehat{f}_{h}(x) f(x) d x+\int f^{2}(x) d x
$$


The ideal choice of bandwidth is the one which minimizes:

$$
L(h)=I S E-\int f^{2}(x) d x=\int \widehat{f}_{h}^{2}(x) d x-2 \int \widehat{f}_{h}(x) f(x) d x .
$$

The principle of the least squares cross-validation method is to find an estimate of $L(h)$ from the data and minimize it over $h$. Consider the estimator

$$
L S C V(h)=\int \widehat{f}_{h}^{2}(x) d x-\frac{2}{n} \sum_{i=1}^{n} \widehat{f}_{h(i)}\left(X_{i}\right)
$$

with

$$
\int \widehat{f}_{h}^{2}(x) d x=\frac{1}{n^{2} h} \sum_{i=1}^{n} \sum_{j=1}^{n}(k * k)\left(\frac{X_{i}-X_{j}}{h}\right)
$$

and

$$
\widehat{f}_{h(i)}\left(X_{i}\right)=\frac{1}{h(n-1)} \sum_{j \neq i}^{n} \mathcal{K}\left(\frac{X_{i}-X_{j}}{h}\right),
$$

where * represents the convolution.

Further discussion on this method can be found in Bowman [3], and Hall and Marron [7]. Under mild conditions, Hall [5] and Stone [22] proved that $h_{L S C V}$ is asymptotically the best in the sense of minimizing $\operatorname{MISE}\left(\widehat{f}_{h}\right)$.

Sheather and Jones [20] introduced a reliable bandwidth selector $\widehat{h}_{S J}$, which is a plug-in estimator of $h_{A M I S E}$, the idea of Sheather and Jones is to estimate the quantity $R\left(f^{\prime \prime}\right)$ by an estimator of $E\left(f^{(4)}(X)\right)$, by note that $R\left(f^{\prime \prime}\right)=E\left(f^{(4)}(X)\right)=\int f^{(4)}(x) f(x) d x$. 
Zhang [26] introduced a generalization of classical least squares cross-validation (LSCV), which provides a significant improvement for LSCV.

Use it as the case that $\mathcal{K}$ is the Gaussian kernel $\phi$. According to Equation (10),

$$
\operatorname{LSCV}(h)=\frac{\phi_{\sqrt{2 h}}(0)}{n}-\frac{2}{n(n-1)} \sum_{i<j}\left[2 \phi_{h}\left(X_{i}-X_{j}\right)+\left(\frac{1}{n}-1\right) \phi_{\sqrt{2 h}}\left(X_{i}-X_{j}\right)\right]
$$

Jin Zhang [26] is the generalized LSCV by:

$$
\begin{aligned}
& \operatorname{LSCV}_{g}(h)=\frac{\phi_{\sqrt{2 h}}(0)}{n} \\
& \quad+\frac{2}{n(n-1)} \sum_{i<j}\left[\frac{2}{g(g-2)} \phi_{\sqrt{g} h}\left(X_{i}-X_{j}\right)+\left(\frac{1}{n}-\frac{1}{g-2}\right) \phi_{\sqrt{2} h}\left(X_{i}-X_{j}\right)\right],
\end{aligned}
$$

$g$ a positive number.

The generalized LSCV bandwidth selector $h_{L S C V}$ is defined as the minimize of $L S C V_{g}(h)$ over $h$.

\section{3. $\beta$-Divergence for Bandwidth Selection}

The basic beta-divergence was introduced by Basu et al. [1] and Minami and Eguchi [14].

The $\beta$-divergence measure for bandwidth selection will be introduced in this section to improve the behaviour of the choice for bandwidth.

$\mathcal{D}_{\beta}\left(\widehat{f}_{h}, f(x)\right)=\frac{1}{\beta} \int_{S} \widehat{f}_{h}^{\beta}(x) d x-\frac{1}{\beta-1} \int_{S} \widehat{f}_{h}^{\beta-1}(x) f(x) d x+\frac{1}{\beta(\beta-1)} \int_{S} f^{\beta}(x) d x$, 
in the case $\beta=2$,

$$
2 \mathcal{D}_{2}\left(\widehat{f}_{h}, f(x)\right)=\operatorname{ISE}\left(\widehat{f}_{h}\right)=\int_{S}\left(\widehat{f}_{h}(x)-f(x)\right)^{2} d x
$$

So we can say that ISE is a special case of $\mathcal{D}_{\beta}$.

The goal of having the window width $h_{\beta}$ which minimizes the mean $\beta$-divergence $\left(E \mathcal{D}_{\beta}\right)$.

$$
\begin{aligned}
& E \mathcal{D}_{\beta}\left(\widehat{f}_{h}, f(x)\right) \\
& \quad=E\left(\frac{1}{\beta} \int_{S} \widehat{f}_{h}^{\beta}(x) d x-\frac{1}{\beta-1} \int_{S} \widehat{f}_{h}^{\beta-1}(x) f(x) d x+\frac{1}{\beta(\beta-1)} \int_{S} f^{\beta}(x) d x\right) .
\end{aligned}
$$

Theorem 1. Let the following conditions on $f$ be satisfied:

(F1) $f$ is compactly supported on I.

(F2) $f$ is four times continuously differentiable on I.

(F3) $\lim _{x \rightarrow+\inf I} f^{(i)}(x)=\lim _{x \rightarrow-\sup I} f^{(i)}(x), 1 \leq j \leq 3$.

(F4) $\int_{I} f^{(2)}(x)^{2} f(x)^{\beta-2} d x<\infty$.

As $n \rightarrow \infty$, the window width $h_{E D_{\beta}}$ that minimizes the mean $\beta$-divergence between a kernel estimator $\widehat{f}_{h}$ and density $f$ satisfies

$$
h_{\beta}=h_{E \mathcal{D}_{\beta}}=\left\{\frac{\int \mathcal{K}(t)^{2} d t \int_{I} f(x)^{\beta-1} d x}{\left[\int t^{2} \mathcal{K}(t) d t\right]^{2} \int_{I} f(x)^{\beta-2} f^{(2)}(x)^{2} d x}\right\}^{1 / 5} n^{-1 / 5}
$$


in the particular case

$-\beta=2$ the mean integrated square error

$$
h_{2}=h_{\operatorname{MISE}\left(\widehat{f}_{h}\right)}=\left\{\frac{\int \mathcal{K}(t)^{2} d t}{\left[\int t^{2} \mathcal{K}(t) d t\right]^{2} \int_{I} f^{(2)}(x)^{2} d x}\right\}^{1 / 5} n^{-1 / 5} .
$$

- $\beta=1$ the Kullback-Leibler,

$$
h_{1}=h_{E(K L)}=\left\{\frac{\int \mathcal{K}(t)^{2} d t \int_{I} d x}{\left[\int t^{2} \mathcal{K}(t) d t\right]^{2} \int_{I} f(x)^{-1} f^{(2)}(x)^{2} d x}\right\}^{1 / 5} n^{-1 / 5} .
$$

Remark 1. For the details about assumptions (F1), (F2), (F3) and (F4), one can refer to [11].

Theorem 1 is derived from the following Proposition 1 by assuming (F4) and by balancing the first two terms in (16).

Proposition 1. Under (F1)-(F3), we have

$$
\begin{aligned}
\operatorname{ED}_{\beta}\left(\widehat{f}_{h}, f\right)= & \frac{h^{4}}{8}\left\{\int_{I} t^{2} \mathcal{K}(t) d t\right\}^{2} \int f(x)^{\beta-2} f^{(2)}(x)^{2} d x \\
& +\frac{1}{2 n h} \int_{I}(\mathcal{K}(t))^{2} d t \int f(x)^{\beta-1} d x+O\left(n^{-1}+h^{6}\right) .
\end{aligned}
$$

Choosing $\beta$ : the $\beta$ value that minimizes Equation (16)

$$
\begin{aligned}
\frac{\partial E \mathcal{D}_{\beta}\left(\widehat{f}_{h}, f\right)}{\partial \beta}= & (\beta-2) \frac{h^{4}}{8}\left\{\int_{I} t^{2} \mathcal{K}(t) d t\right\}^{2} \int f(x)^{\beta-3} f^{(2)}(x)^{2} d x \\
& +\frac{\beta-1}{2 n h} \int_{I}(\mathcal{K}(t))^{2} d t \int f(x)^{\beta-2} d x+O\left(n^{-1}+h^{6}\right),
\end{aligned}
$$

we $\quad$ pose $\left.\quad \Delta_{1}=\frac{h^{4}}{8} \iint_{I} t^{2} \mathcal{K}(t) d t\right\}^{2} \int f(x)^{\beta-3} f^{(2)}(x)^{2} d x \quad$ and $\quad \Delta_{2}=\frac{1}{2 n h} \int_{I}$ $(\mathcal{K}(t))^{2} d t \int f(x)^{\beta-2} d x$ 


$$
\begin{gathered}
\frac{\partial E \mathcal{D}_{\beta}\left(\widehat{f}_{h}, f\right)}{\partial \beta}=(\beta-2) \Delta_{1}+(\beta-1) \Delta_{2}=0, \\
\beta=1+\frac{\Delta_{1}}{\Delta_{1}+\Delta_{2}},
\end{gathered}
$$

we know that $\Delta_{1} \geq 0$ and $\Delta_{2} \geq 0$. This implies that:

$$
1<\beta<2 \text {. }
$$

\section{Proof of Proposition 1.}

With a random variable $\xi=O_{p}(1)$ whose expectation is 0 and variance 1 , we can write $\widehat{f}_{h}(x)$ as (see, [11])

$$
\begin{aligned}
\widehat{f}_{h}(x)= & f(x)\left[1+\frac{h^{2}}{2} \frac{f^{(2)}(x)}{f(x)} \int_{I} t^{2} \mathcal{K}(t) d t+\frac{h^{4}}{24} \frac{f^{(4)}(x)}{f(x)} \int_{I} t^{4} \mathcal{K}(t) d t\right. \\
& \left.+O\left(h^{6}\right)+\left\{\frac{\int_{I} \mathcal{K}(t)^{2} d t}{n h f(x)}\right\} \xi+O_{p}\left(n^{-1 / 2}\right)\right],
\end{aligned}
$$

where the $O\left(h^{6}\right)$ terms depend upon $x$. Using $(1+z)^{\beta}=1+\beta z+$ $\frac{\beta(\beta-1)}{2} z^{2}+O\left(z^{3}\right)$

$\widehat{f}_{h}^{\beta}(x)=f^{\beta}(x)[1+\beta$

$$
\begin{aligned}
& \left(\frac{1}{2} h^{2} \frac{f^{(2)}(x)}{f(x)} \int_{I} t^{2} \mathcal{K}(t) d t+\frac{1}{24} h^{4} \frac{f^{(4)}(x)}{f(x)} \int_{I} t^{4} \mathcal{K}(t) d t+\left\{\frac{\int_{I} \mathcal{K}(t)^{2} d t}{n h f(x)}\right\}^{1 / 2} \quad \xi\right) \\
& \left.+\frac{\beta(\beta-1)}{2}\left[\frac{1}{4} h^{4} \frac{f^{(2)}(x)^{2}}{f(x)^{2}}\left(\int_{I} t^{2} \mathcal{K}(t) d t\right)^{2}+\frac{\int_{I} \mathcal{K}(t)^{2} d t}{n h f(x)} \xi^{2}\right]+O\left(h^{6}\right)+O_{p}\left(n^{-1 / 2}\right)\right],
\end{aligned}
$$




$$
\begin{aligned}
& \widehat{f}_{h}^{\beta-1}(x)=f^{\beta-1}(x)[1+(\beta-1) \\
& \left(\frac{1}{2} h^{2} \frac{f^{(2)}(x)}{f(x)} \int_{I} t^{2} \mathcal{K}(t) d t+\frac{1}{24} h^{4} \frac{f^{(4)}(x)}{f(x)} \int_{I} t^{4} \mathcal{K}(t) d t+\left\{\frac{\int_{I} \mathcal{K}(t)^{2} d t}{n h f(x)}\right\}^{1 / 2} \xi\right) \\
& +\frac{(\beta-1)(\beta-2)}{2}\left[\frac{1}{4} h^{4} \frac{f^{(2)}(x)^{2}}{f(x)^{2}}\left(\int_{I} t^{2} \mathcal{K}(t) d t\right)^{2}+\frac{\int_{I} \mathcal{K}(t)^{2} d t}{n h f(x)} \xi^{2}\right] \\
& \left.+O\left(h^{6}\right)+O_{p}\left(n^{-1 / 2}\right)\right], \\
& \mathcal{D}_{\beta}\left(\widehat{f}_{h}, f\right)=\int f(x)^{\beta}\left[\frac{1}{\beta}-\frac{1}{\beta-1}+\left(\frac{\beta-1}{2}-\frac{\beta-2}{2}\right)\left[\frac{1}{4} h^{4} \frac{f^{(2)}(x)^{2}}{f(x)^{2}}\left(\int_{I} t^{2} \mathcal{K}(t) d t\right)^{2}\right.\right. \\
& \left.\left.+\frac{\int_{I} \mathcal{K}(t)^{2} d t}{n h f(x)} \xi^{2}\right]+O\left(h^{2}\right)+O_{p}\left(n^{-1 / 2}\right)\right] d x+\frac{1}{\beta(\beta-1)} \int f(x)^{\beta} d x \\
& =\frac{1}{2} \int f(x)^{\beta}\left[\frac{1}{4} h^{4} \frac{f^{(2)}(x)^{2}}{f(x)^{2}}\left(\int_{I} t^{2} \mathcal{K}(t) d t\right)^{2}+\frac{\int_{I} \mathcal{K}(t)^{2} d t}{n h f(x)} \xi^{2}+O\left(h^{6}\right)+O_{p}\left(n^{-1 / 2}\right)\right] d x,
\end{aligned}
$$

the last term vanishes upon taking expectations for $E\left(\xi^{2}\right)=0$. Hence we obtain

$$
\begin{aligned}
& \operatorname{ED}_{\beta}\left(\widehat{f}_{h}, f\right)=\frac{1}{2} E \int f(x)^{\beta} \\
& {\left[\frac{1}{4} h^{4} \frac{f^{(2)}(x)^{2}}{f(x)^{2}}\left(\int_{I} t^{2} \mathcal{K}(t) d t\right)^{2}+\frac{\int_{I} \mathcal{K}(t)^{2} d t}{n h f(x)} \xi^{2}+O\left(h^{6}\right)+O_{p}\left(n^{-1 / 2}\right)\right] d x}
\end{aligned}
$$




$$
\begin{aligned}
=\frac{h^{4}}{8}\left\{\int_{I} t^{2} \mathcal{K}(t) d t\right\}^{2} \int & f(x)^{\beta-2} f^{(2)}(x)^{2} d x \\
& +\frac{1}{2 n h} \int_{I}(\mathcal{K}(t))^{2} d t \int f(x)^{\beta-1} E\left(\xi^{2}\right) d x+O\left(n^{-1}+h^{6}\right),
\end{aligned}
$$

we know that $E\left(\xi^{2}\right)=1$

$$
\begin{aligned}
\operatorname{ED}_{\beta}\left(\widehat{f}_{h}, f\right)= & \frac{h^{4}}{8}\left\{\int_{I} t^{2} \mathcal{K}(t) d t\right\}^{2} \int f(x)^{\beta-2} f^{(2)}(x)^{2} d x \\
& +\frac{1}{2 n h} \int_{I}(\mathcal{K}(t))^{2} d t \int f(x)^{\beta-1} d x+O\left(n^{-1}+h^{6}\right)
\end{aligned}
$$

as required.

\section{Simulation}

We approximate the true density $f$ by a normal mixture

$$
f(x)=\sum_{j=1}^{J} \omega_{j} \phi_{\sigma_{j}}\left(x-\mu_{j}\right),
$$

where $J$ is a positive integer. $\omega_{1}, \ldots, \omega_{j}$ is a set of positive numbers that sum to one, and for each $j,-\infty<\mu_{j}<\infty$ and $\sigma_{j}>0$. The family of normal mixture densities used by Marron and Wand [13] is extremely rich, and, in fact, any density can be approximated arbitrarily well by a member of this family, for $f$ given by Equation (23), the MISE in Equation (2) of the kernel density estimator in Equation (1) have explicit forms. In fact,

$$
\operatorname{MISE}\left(\widehat{f}_{h}(x)\right)=\left(2 \pi^{1 / 2} n h\right)^{-1}+w^{T}\left\{\left(1-n^{-1}\right) \Omega_{2}-2 \Omega_{1}+\Omega_{0}\right\} w,
$$

(Marron and Wand [13]) where $w=\left(\omega_{1}, \ldots, \omega_{J}\right)$ and $\Omega_{a}$ is the $J \times J$ matrix having $(j \times j)$ entry equal to

$$
\phi \sqrt{a h^{2}+\sigma_{j}^{2}+\sigma_{j^{\prime}}^{2}}\left(\mu_{j}-\mu_{j^{\prime}}\right) .
$$


We consider the normal mixture in the case of $J=2$ and $\omega_{1}=\omega_{2}=0.5$, similar simulation were performed by [26]. Therefore, the true density is:

$$
f(x)=0.5 \phi(x)+0.5 \phi_{\sigma}(x-\mu) .
$$

Based on 50, 200, 700 draws from $f$ in the case where $\mu=0,1,5$ and $\sigma=1,0.5,0.1$. Table 1 give the exhibits simulated relative efficiency $\operatorname{RE}(\widehat{h})=\operatorname{MISE}\left(\widehat{f}_{\widehat{h}_{M I S E}}\right) / \operatorname{MISE}\left(\widehat{f}_{\widehat{h}}\right)$ of the kernel estimator, using bandwidths $\widehat{h}_{N R}, \widehat{h}_{L S C V}, \widehat{h}_{S J}, \widehat{h}_{L S C V_{4}}$, and $\widehat{h}_{E \mathcal{D}_{\beta}}$ (with $\beta=1.1,1.5$, and 1.8), it is lower than 1 , because the optimal bandwidth $h_{M I S E}$ minimize MISE. Each bandwidth, mean $E(\widehat{h})$ and mean relation error $E\left|\widehat{h} / h_{M I S E}-1\right|$ are obtained, these values are given by respectively, Tables 2 and 3. 
Table 1. $R E(\widehat{h})$ for normal mixture $f(x)=0.5 \phi(x)+0.5 \phi_{\sigma}(x-\mu)$

\begin{tabular}{|c|c|c|c|c|c|c|c|}
\hline$N$ & $\widehat{h}_{N R}$ & $\widehat{h}_{L S C V}$ & $\widehat{h}_{S J}$ & $\widehat{h}_{L S C V_{4}}$ & $\widehat{h}_{\mathcal{D}_{1.1}}$ & $\widehat{h}_{\mathcal{D}_{1.5}}$ & $\widehat{h}_{\mathcal{D}_{1.8}}$ \\
\hline & & & $\mu=0$ & $\sigma=1$ & & & \\
\hline 50 & 0.877 & 0.633 & 0.798 & 0.909 & 0.768 & 0.716 & 0.694 \\
\hline 200 & 0.928 & 0.730 & 0.867 & 0.944 & 0.978 & 0.944 & 0.924 \\
\hline \multirow[t]{2}{*}{700} & 0.974 & 0.767 & 0.942 & 0.956 & 0.978 & 0.981 & 0.973 \\
\hline & & & $\mu=0$ & $\sigma=0.5$ & & & \\
\hline 50 & 0899 & 0.633 & 0.850 & 0.776 & 0.600 & 0.900 & 0.917 \\
\hline 200 & 0.948 & 0.644 & 0.895 & 0.908 & 0.884 & 0.987 & 0.949 \\
\hline \multirow[t]{2}{*}{700} & 0.952 & 0.767 & 0.939 & 0.914 & 0.980 & 0.821 & 0.816 \\
\hline & & & $\mu=0$ & $\sigma=0.1$ & & & \\
\hline 50 & 0.562 & 0.781 & 0.779 & 0.748 & 0.700 & 0.618 & 0.573 \\
\hline 200 & 0.550 & 0.810 & 0.902 & 0.891 & 0.930 & 0.890 & 0.747 \\
\hline \multirow[t]{2}{*}{700} & 0.535 & 0.872 & 0.966 & 0.957 & 0.993 & 0.977 & 0.963 \\
\hline & & & $\mu=1$ & $\sigma=1$ & & & \\
\hline 50 & 0.871 & 0.585 & 0.791 & 0.904 & 0.665 & 0.830 & 0.855 \\
\hline 200 & 0.955 & 0.699 & 0.905 & 0.949 & 0.928 & 0.989 & 0.992 \\
\hline \multirow[t]{2}{*}{700} & 0.977 & 0.763 & 0.943 & 0.960 & 0.981 & 0.973 & 0.977 \\
\hline & & & $\mu=1$ & $\sigma=0.5$ & & & \\
\hline 50 & 0.855 & 0.562 & 0.856 & 0.806 & 0.749 & 0.751 & 0.906 \\
\hline 200 & 0.867 & 0.760 & 0.929 & 0.874 & 0.965 & 0.975 & 0.989 \\
\hline \multirow[t]{2}{*}{700} & 0.823 & 0.831 & 0.955 & 0.922 & 0.942 & 0.923 & 0.720 \\
\hline & & & $\mu=1$ & $\sigma=0.1$ & & & \\
\hline 50 & 0.2370 & 0.781 & 0.439 & 0.755 & 0.544 & 0.606 & 0.531 \\
\hline 200 & 0.1010 & 0.810 & 0.419 & 0.904 & 0.756 & 0.834 & 0.717 \\
\hline \multirow[t]{2}{*}{700} & 0.0503 & 0.912 & 0.557 & 0.949 & 0.968 & 0.980 & 0.921 \\
\hline & & & $\mu=5$ & $\sigma=1$ & & & \\
\hline 50 & 0.411 & 0.741 & 0.875 & 0.864 & 0.618 & 0.883 & 0.490 \\
\hline 200 & 0.285 & 0.861 & 0.947 & 0.948 & 0.852 & 0.981 & 0.688 \\
\hline 700 & 0.215 & 0.876 & 0.972 & 0.967 & 0.997 & 0.870 & 0.914 \\
\hline
\end{tabular}


Table 1. (Continued)

\begin{tabular}{cccccccc}
\hline$N$ & $\widehat{h}_{N R}$ & $\widehat{h}_{L S C V}$ & $\widehat{h}_{S J}$ & $\widehat{h}_{L S C V_{4}}$ & $\widehat{h}_{\mathcal{D}_{1.1}}$ & $\widehat{h}_{\mathcal{D}_{1.5}}$ & $\widehat{h}_{\mathcal{D}_{1.8}}$ \\
\hline & & & $\mu=5$ & $\sigma=0.5$ & & & \\
50 & 0.2390 & 0.707 & 0.696 & $\mathbf{0 . 8 7 1}$ & 0.657 & $\mathbf{0 . 8 7 1}$ & $\mathbf{0 . 8 0 6}$ \\
200 & 0.1330 & 0.760 & 0.747 & $\mathbf{0 . 9 2 3}$ & $\mathbf{0 . 9 0 7}$ & $\mathbf{0 . 9 8 6}$ & $\mathbf{0 . 9 8 3}$ \\
700 & 0.0804 & $\mathbf{0 . 8 4 2}$ & $\mathbf{0 . 8 4 6}$ & $\mathbf{0 . 9 5 7}$ & $\mathbf{0 . 9 9 0}$ & $\mathbf{0 . 8 0 0}$ & $\mathbf{0 . 9 1 2}$ \\
& & & $\mu=5$ & $\sigma=0.1$ & & & \\
50 & 0.1360 & 0.588 & 0.1760 & 0.791 & 0.630 & 0.563 & 0.693 \\
200 & 0.0523 & 0.458 & 0.0946 & $\mathbf{0 . 9 0 5}$ & $\mathbf{0 . 8 7 8}$ & 0.774 & $\mathbf{0 . 9 4 0}$ \\
700 & 0.0205 & 0.341 & 0.0665 & $\mathbf{0 . 9 6 9}$ & $\mathbf{0 . 9 6 8}$ & $\mathbf{0 . 9 5 5}$ & $\mathbf{0 . 9 8 9}$ \\
\hline
\end{tabular}


Table 2. $E(\widehat{h})$ for normal mixture $f(x)=0.5 \phi(x)+0.5 \phi_{\sigma}(x-\mu)$

\begin{tabular}{|c|c|c|c|c|c|c|c|c|}
\hline$N$ & $\widehat{h}_{N R}$ & $\widehat{h}_{L S C V}$ & $\widehat{h}_{S J}$ & $\widehat{h}_{L S C V_{4}}$ & $\widehat{h}_{\mathcal{D}_{1.1}}$ & $\widehat{h}_{\mathcal{D}_{1.5}}$ & $\widehat{h}_{\mathcal{D}_{1.8}}$ & $h_{M I S E}$ \\
\hline & & & & $\mu=0$ & $\sigma=1$ & & & \\
\hline 50 & 0.455 & 0.480 & 0.452 & 0.516 & 0.323 & 0.330 & 0.379 & 0.520 \\
\hline 200 & 0.354 & 0.373 & 0.349 & 0.385 & 0.321 & 0.328 & 0.376 & 0.383 \\
\hline \multirow[t]{2}{*}{700} & 0.283 & 0.288 & 0.282 & 0.301 & 0.308 & 0.309 & 0.307 & 0.293 \\
\hline & & & & $\mu=0$ & $\sigma=0.5$ & & & \\
\hline 50 & 0.318 & 0.362 & 0.306 & 0.382 & 0.223 & 0.286 & 0.286 & 0.343 \\
\hline 200 & 0.250 & 0.262 & 0.231 & 0.266 & 0.193 & 0.280 & 0.285 & 0.248 \\
\hline \multirow[t]{2}{*}{700} & 0.197 & 0.191 & 0.184 & 0.200 & 0.186 & 0.244 & 0.245 & 0.188 \\
\hline & & & & $\mu=0$ & $\sigma=0.1$ & & & \\
\hline 50 & 0.1320 & 0.0897 & 0.0915 & 0.1040 & 0.510 & 0.0409 & 0.0356 & 0.0752 \\
\hline 200 & 0.0887 & 0.0557 & 0.0572 & 0.0597 & 0.485 & 0.0377 & 0.0331 & 0.0530 \\
\hline \multirow[t]{2}{*}{700} & 0.0668 & 0.0406 & 0.0406 & 0.0422 & 0.421 & 0.0370 & 0.0329 & 0.0398 \\
\hline & & & & $\mu=1$ & $\sigma=1$ & & & \\
\hline 50 & 0.510 & 0.569 & 0.511 & 0.573 & 0.429 & 0.426 & 0.435 & 0.373 \\
\hline 200 & 0.403 & 0.418 & 0.403 & 0.440 & 0.395 & 0.423 & 0.429 & 0.265 \\
\hline \multirow[t]{2}{*}{700} & 0.317 & 0.329 & 0.315 & 0.337 & 0.354 & 0.345 & 0.346 & 0.199 \\
\hline & & & & $\mu=1$ & $\sigma=0.5$ & & & \\
\hline 50 & 0.409 & 0.395 & 0.366 & 0.446 & 0.326 & 0.342 & 0.283 & 0.373 \\
\hline 200 & 0.328 & 0.278 & 0.270 & 0.296 & 0.280 & 0.282 & 0.283 & 0.265 \\
\hline \multirow[t]{2}{*}{700} & 0.256 & 0.197 & 0.203 & 0.212 & 0.233 & 0.239 & 0.281 & 0.199 \\
\hline & & & & $\mu=1$ & $\sigma=0.1$ & & & \\
\hline 50 & 0.351 & 0.0877 & 0.1780 & 0.1030 & 0.0422 & 0.0451 & 0.0523 & 0.0752 \\
\hline 200 & 0.280 & 0.0553 & 0.1050 & 0.0614 & 0.0380 & 0.0380 & 0.0401 & 0.0530 \\
\hline \multirow[t]{2}{*}{700} & 0.222 & 0.0408 & 0.0657 & 0.0426 & 0.0343 & 0.0314 & 0.0311 & 0.0398 \\
\hline & & & & $\mu=5$ & $\sigma=1$ & & & \\
\hline 50 & 1.300 & 0.636 & 0.753 & 0.742 & 0.420 & 0.475 & 0.315 & 0.608 \\
\hline 200 & 0.986 & 0.456 & 0.495 & 0.472 & 0.330 & 0.470 & 0.285 & 0.441 \\
\hline 700 & 0.770 & 0.342 & 0.361 & 0.261 & 0.442 & 0.272 & 0.276 & 0.336 \\
\hline
\end{tabular}


Table 2. (Continued)

\begin{tabular}{ccccccccc}
\hline$N$ & $\widehat{h}_{N R}$ & $\widehat{h}_{L S C V}$ & $\widehat{h}_{S J}$ & $\widehat{h}_{L S C V_{4}}$ & $\widehat{h}_{\mathcal{D}_{1.1}}$ & $\widehat{h}_{\mathcal{D}_{1.5}}$ & $\widehat{h}_{\mathcal{D}_{1.8}}$ & $h_{M I S E}$ \\
\hline & & & & $\mu=5$ & $\sigma=0.5$ & & & \\
50 & 1.260 & 0.407 & 0.590 & 0.448 & 0.310 & $\mathbf{0 . 2 9 5}$ & $\mathbf{0 . 2 5 1}$ & $\mathbf{0 . 3 6 9}$ \\
200 & 0.963 & $\mathbf{0 . 2 7 4}$ & $\mathbf{0 . 3 7 8}$ & $\mathbf{0 . 2 9 6}$ & $\mathbf{0 . 2 1 0}$ & $\mathbf{0 . 2 8 6}$ & $\mathbf{0 . 2 5 0}$ & $\mathbf{0 . 2 6 2}$ \\
700 & 0.750 & $\mathbf{0 . 2 0 1}$ & $\mathbf{0 . 2 5 5}$ & $\mathbf{0 . 2 1 0}$ & $\mathbf{0 . 2 0 9}$ & $\mathbf{0 . 2 7 0}$ & $\mathbf{0 . 2 4 4}$ & $\mathbf{0 . 1 9 7}$ \\
& & & & $\mu=5$ & $\sigma=0.1$ & & & \\
50 & 1.260 & $\mathbf{0 . 0 8 7 1}$ & 0.523 & $\mathbf{0 . 1 0 4 0}$ & $\mathbf{0 . 0 4 5}$ & $\mathbf{0 . 0 4 1 5}$ & $\mathbf{0 . 0 4 7 6}$ & $\mathbf{0 . 0 7 5 2}$ \\
200 & 0.956 & $\mathbf{0 . 0 5 6 0}$ & $\mathbf{0 . 2 9 2}$ & $\mathbf{0 . 0 6 0 3}$ & $\mathbf{0 . 0 4 0}$ & $\mathbf{0 . 0 3 8 5}$ & $\mathbf{0 . 0 4 2 7}$ & $\mathbf{0 . 0 5 3 0}$ \\
700 & 0.745 & $\mathbf{0 . 0 4 0 6}$ & $\mathbf{0 . 1 7 2}$ & $\mathbf{0 . 0 4 2 0}$ & $\mathbf{0 . 0 3 9}$ & $\mathbf{0 . 0 3 3 9}$ & $\mathbf{0 . 0 4 2 6}$ & $\mathbf{0 . 0 3 9 8}$ \\
\hline
\end{tabular}


Table 3. $E\left|\widehat{h} / h_{M I S E}-1\right|$ for normal mixture $f(x)=0.5 \phi(x)+0.5 \phi_{\sigma}(x-\mu)$

\begin{tabular}{|c|c|c|c|c|c|c|c|}
\hline$N$ & $\widehat{h}_{N R}$ & $\widehat{h}_{L S C V}$ & $\widehat{h}_{S J}$ & $\widehat{h}_{L S C V_{4}}$ & $\widehat{h}_{\mathcal{D}_{1.1}}$ & $\widehat{h}_{\mathcal{D}_{1.5}}$ & $\widehat{h}_{\mathcal{D}_{1.8}}$ \\
\hline & & & $\mu=0$ & $\sigma=1$ & & & \\
\hline 50 & 0.1350 & 0.1240 & 0.1640 & 0.0874 & 0.3790 & 0.3650 & 0.2710 \\
\hline 200 & 0.0785 & 0.0829 & 0.1050 & 0.0578 & 0.1620 & 0.1390 & 0.0177 \\
\hline \multirow[t]{2}{*}{700} & 0.0396 & 0.0717 & 0.0572 & 0.0436 & 0.0509 & 0.0531 & 0.0486 \\
\hline & & & $\mu=0$ & $\sigma=0.5$ & & & \\
\hline 50 & 0.1370 & 0.1510 & 0.1670 & 0.1510 & 0.4560 & 0.179 & 0.166 \\
\hline 200 & 0.0655 & 0.1360 & 0.0882 & 0.1010 & 0.2490 & 0.135 & 0.150 \\
\hline \multirow[t]{2}{*}{700} & 0.0559 & 0.0729 & 0.0537 & 0.0818 & 0.0104 & 0.112 & 0.132 \\
\hline & & & $\mu=0$ & $\sigma=0.1$ & & & \\
\hline 50 & 0.772 & 0.2530 & 0.3000 & 0.3990 & 0.4410 & 0.4980 & 0.562 \\
\hline 200 & 0.674 & 0.1210 & 0.1250 & 0.1520 & 0.2070 & 0.2870 & 0.378 \\
\hline \multirow[t]{2}{*}{700} & 0.679 & 0.0772 & 0.0506 & 0.0726 & 0.185 & 0.0503 & 0.171 \\
\hline & & & $\mu=1$ & $\sigma=1$ & & & \\
\hline 50 & 0.1430 & 0.1040 & 0.1630 & 0.0748 & 0.398 & 0.2760 & 0.2610 \\
\hline 200 & 0.0774 & 0.0800 & 0.0931 & 0.0501 & 0.184 & 0.0249 & 0.0124 \\
\hline \multirow[t]{2}{*}{700} & 0.0483 & 0.0626 & 0.0600 & 0.0361 & 0.037 & 0.0426 & 0.0416 \\
\hline & & & $\mu=1$ & $\sigma=0.5$ & & & \\
\hline 50 & 0.172 & 0.1930 & 0.1400 & 0.2260 & 0.4560 & 0.3580 & 0.2420 \\
\hline 200 & 0.236 & 0.1530 & 0.0899 & 0.1460 & 0.121 & 0.0965 & 0.0668 \\
\hline \multirow[t]{2}{*}{700} & 0.285 & 0.0989 & 0.0506 & 0.0794 & 0.119 & 0.1010 & 0.0400 \\
\hline & & & $\mu=1$ & $\sigma=0.1$ & & & \\
\hline 50 & 3.67 & 0.2620 & 1.380 & 0.3980 & 0.544 & 0.507 & 0.586 \\
\hline 200 & 4.29 & 0.1250 & 0.986 & 0.1720 & 0.353 & 0.300 & 0.413 \\
\hline \multirow[t]{2}{*}{700} & 4.58 & 0.0838 & 0.652 & 0.0878 & 0.137 & 0.068 & 0.218 \\
\hline & & & $\mu=5$ & $\sigma=1$ & & & \\
\hline 50 & 1.14 & 0.1450 & 0.2390 & 0.2430 & 0.4580 & 0.2840 & 0.570 \\
\hline 200 & 1.23 & 0.0815 & 0.1210 & 0.0899 & 0.2530 & 0.0147 & 0.409 \\
\hline 700 & 1.29 & 0.0686 & 0.0745 & 0.0540 & 0.0203 & 0.2970 & 0.224 \\
\hline
\end{tabular}


Table 3. (Continued)

\begin{tabular}{cccccccc}
\hline \multirow{2}{*}{$N$} & $\widehat{h}_{N R}$ & $\widehat{h}_{L S C V}$ & $\widehat{h}_{S J}$ & $\widehat{h}_{L S C V_{4}}$ & $\widehat{h}_{\mathcal{D}_{1.1}}$ & $\widehat{h}_{\mathcal{D}_{1.5}}$ & $\widehat{h}_{\mathcal{D}_{1.8}}$ \\
\hline & & & $\mu=5$ & $\sigma=0.5$ & & & \\
50 & 2.40 & $\mathbf{0 . 1 8 6 0}$ & 0.600 & $\mathbf{0 . 2 5 1 0}$ & 0.4340 & $\mathbf{0 . 2 6 6 0}$ & 0.3380 \\
200 & 2.68 & $\mathbf{0 . 1 1 8 0}$ & 0.444 & $\mathbf{0 . 1 4 4 0}$ & $\mathbf{0 . 2 0 2 0}$ & $\mathbf{0 . 0 3 1 5}$ & $\mathbf{0 . 0 6 7 3}$ \\
700 & 2.80 & $\mathbf{0 . 0 7 4 3}$ & $\mathbf{0 . 2 9 6}$ & $\mathbf{0 . 0 8 0 4}$ & $\mathbf{0 . 0 5 9 7}$ & $\mathbf{0 . 0 3 0 0}$ & $\mathbf{0 . 0 4 9 0}$ \\
& & & $\mu=5$ & $\sigma=0.1$ & & & \\
50 & 15.7 & 0.884 & 5.95 & 0.3980 & 0.4810 & 0.549 & 0.433 \\
200 & 17.1 & 1.021 & 4.51 & $\mathbf{0 . 1 5 7 0}$ & $\mathbf{0 . 2 6 3 0}$ & 0.359 & $\mathbf{0 . 1 9 4}$ \\
700 & 17.7 & 1.104 & 3.34 & $\mathbf{0 . 0 6 5 6}$ & $\mathbf{0 . 0 1 7 8}$ & $\mathbf{0 . 1 4 6}$ & $\mathbf{0 . 0 7 4}$ \\
\hline
\end{tabular}

(1) It can be seen that when the density $f$ is not very far from normal, with $(\mu, \sigma)=(0,1),(0,0.5),(1,1)$, and $(1,0.5)$, bandwidth which are obtained by using NR criterion performs well. In other cases, it usually has the smallest $R E(\widehat{h})$ and largest $E(\widehat{h})$, tending to over smooth its kernel density estimate the most.

(2) From these tables, it can be seen that bandwidth $\widehat{h}_{L S C V}$ which are obtained by using LSCV performs poorly if the true density is close to normal usually having the smallest $R E(\widehat{h})$ and $E(\widehat{h})$, then the kernel density estimator is undersmoothed. But in the contrary case, $\widehat{h}_{L S C V}$ can perform very well, and in many situations, in Table 2 , it is seen that $E\left(\widehat{h}_{L S C V}\right)$ is close to the optimal $\widehat{h}_{M I S E}$, but the corresponding $E\left(\widehat{h}_{L S C V} / \widehat{h}_{M I S E}-1\right)$ is large, which means that the bias of $\widehat{h}_{L S C V}$ is small but its variation is large.

(3) The plug-in bandwidth $\widehat{h}_{S J}$ seen to be the best existing bandwidth selectors (as commented by [23]). $\widehat{h}_{S J}$ among the best bandwidth selectors in most situations. However it behaves very poorly for small $\sigma$ (the density curve is sharp), oversmoothing its kernel density curve by overestimating $\widehat{h}_{M I S E}$. 
(4) As commented by Zhang [26], the generalized LSCV bandwidth $\widehat{h}_{L_{S C V_{4}}}$ seems to be bandwidth selectors that is always among the best for having large $R E(\widehat{h})$ and small $E\left|\widehat{h} / h_{M I S E}-1\right|$.

(5) The proposed bandwidth selector method $\left(\widehat{h}_{\mathcal{D}_{\beta}}\right)$ dominate the selection methods bandwidth as $n$ increases, otherwise, $\widehat{h}_{\mathcal{D}_{\beta}}$ (with $\beta=1.1,1.5$, and 1.8) are with $\widehat{h}_{L S C V_{4}}$ bandwidths selectors are always among the best for having large $R E\left(\widehat{h}_{\mathcal{D}_{\beta}}\right)$ and small $E\left|\widehat{h}_{\mathcal{D}_{\beta}} / h_{M I S E}-1\right|$ most cases. They significantly improve the classical $\widehat{h}_{L S C V}$. Indeed, Figure 1 shows that the increase in $n$ causes a value of $R E\left(\widehat{h}_{\mathcal{D}_{\beta}}\right)$ close to 1 and greater than $R E\left(\widehat{h}_{L S C V}\right)$.

Figure 1 shows that for the $\mathcal{D}_{\beta}$, this bias does not have a serious effect on the efficiency of the method, since the $R E\left(\widehat{h}_{\mathcal{D}_{\beta}}\right)$ is relatively close to 1 . We can conclude from Figure 1 our practical selection procedures $\mathcal{D}_{\beta}$ have a performance close to the one for the $L S C V_{4}$ bandwidth selector (since the ratios are relatively close to 1). 
$\mu=0$ and $\sigma=1$

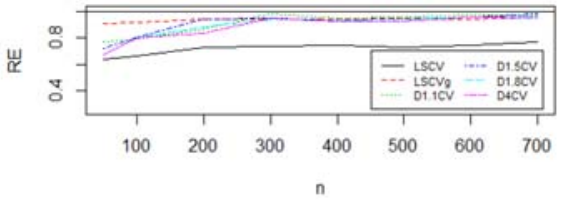

$\mu=0$ and $\sigma=0.5$

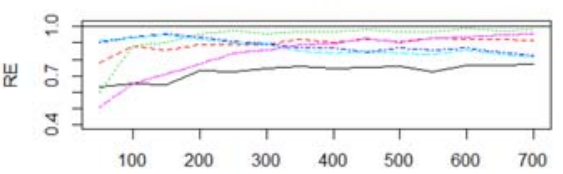

$\mu=1$ and $\sigma=0.5$

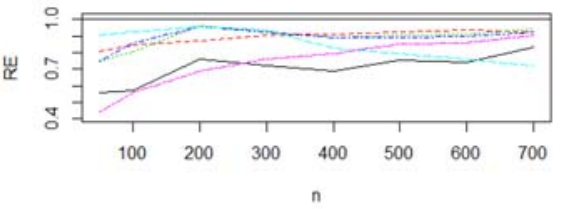

$\mu=1$ and $\sigma=0.1$

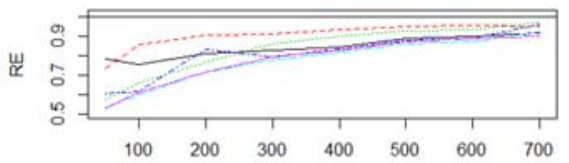

$\mu=5$ and $\sigma=0.1$

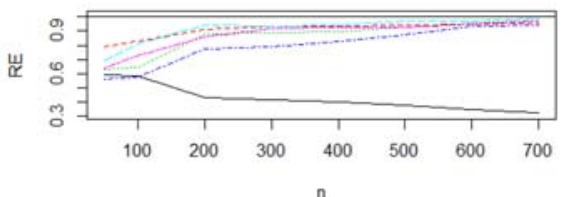

$\mu=0$ and $\sigma=0.1$

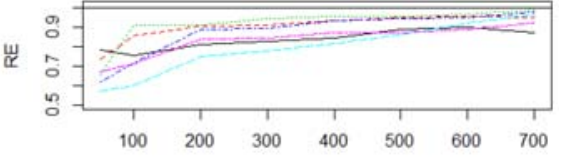

$\mu=1$ and $\sigma=1$

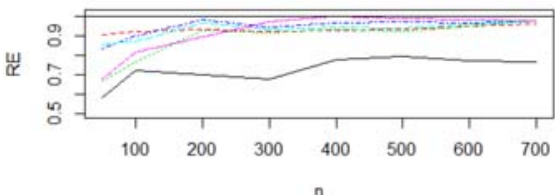

$\mu=5$ and $\sigma=1$
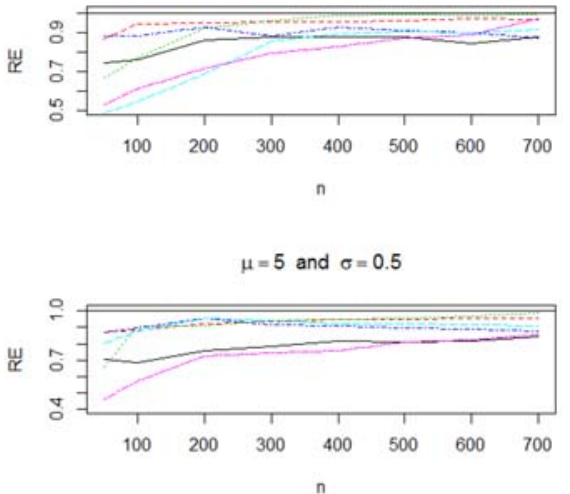

Figure 1. $R E(\widehat{h})$ using bandwidths $\widehat{h}_{L S C V}, \widehat{h}_{L S C V_{4}}$, and $\widehat{h}_{\mathcal{D}_{\beta}}$ with $\beta=1.1,1.5,1.8$. 


\section{Examples}

In this section, we will provided two examples to evaluate performance of our method compared to several classical bandwidth selection methods for Gaussian kernel density. The two data sets in the examples have been analyzed by many authors ([21] and [26]) to illustrate various kinds of methods in density estimation.

The first example comprises the lengths of 86 spells of psychiatric treatment undergone by control patients used as controls in a study of suicide risks reported by Fryer [4] and Silverman [21].

Figure 2(a) plot the data points and the kernel density estimates for the suicide study data, when we using commonly used bandwidths $\widehat{h}_{N R}=35.78$, $\widehat{h}_{S J}=23.16, \widehat{h}_{L S C V}=15.69, \widehat{h}_{L_{S C V_{4}}}=22.57$, and $\widehat{h}_{\mathcal{D}_{1.1}}=24.25$.

It seems that the density for the length of treatment is a unimodal curve heavily skewed to the right, it seems clear than the bandwidth $\widehat{h}_{N R}$ oversmooth her kernel density curve and underestimate the peak near 20 .

The bandwidths were obtained by using the methods of $S J, L S C V_{4}$, and $\mathcal{D}_{1.1}$, are better because $\widehat{h}_{S J}, \widehat{h}_{L S C V_{4}}$, and $\widehat{h}_{\mathcal{D}_{1.1}}$ well balance the two situations and seem to capture the true shape of the data. In this example, $\widehat{h}_{L_{S C V_{4}}}=27.57$ and $\widehat{h}_{\mathcal{D}_{1.1}}=24.25$ are closer to $\widehat{h}_{S J}=23.16$.

The second comprises the lengths in minutes of 107 eruption lengths in minutes for the old faithful geyser in Yellowstone National Park, USA (source: Weisberg [25] and Silvermanl [21]).

Figure 2(b) plot the data points and the kernel density estimates for old faithful geyser data, we using bandwidths $\widehat{h}_{N R}=0.4331, \widehat{h}_{S J}=0.2250$, $\widehat{h}_{L S C V}=0.1030, \widehat{h}_{L_{S C V_{4}}}=0.1740$, and $\widehat{h}_{\mathcal{D}_{1.1}}=0.1480$. 
(a) Densities of lengths of treatment of control patients in suicide

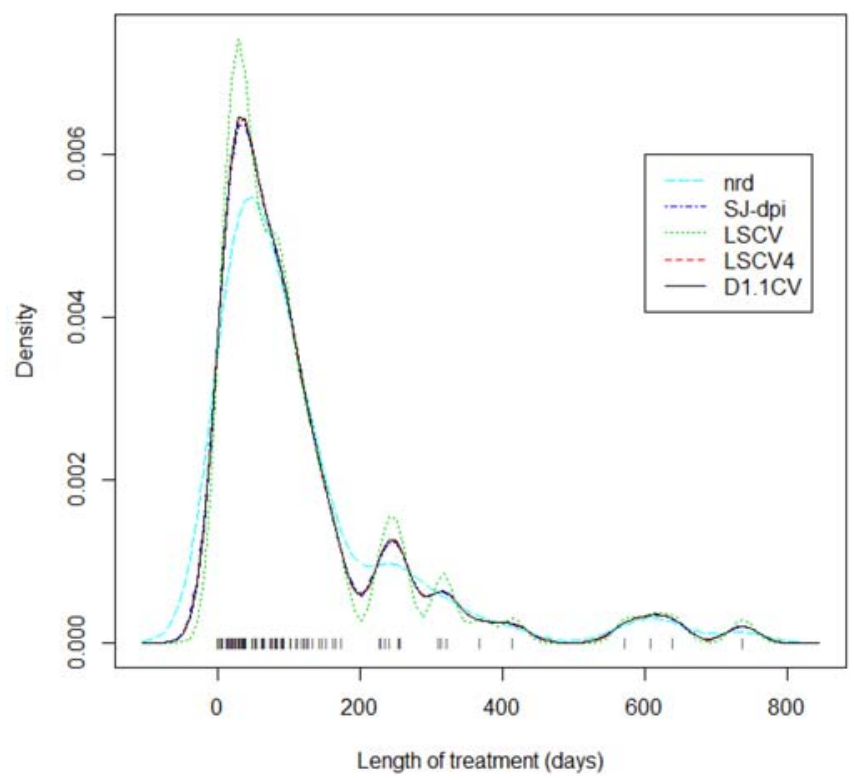

(b) Densities of eruption lengths of Old Faithful geyser.

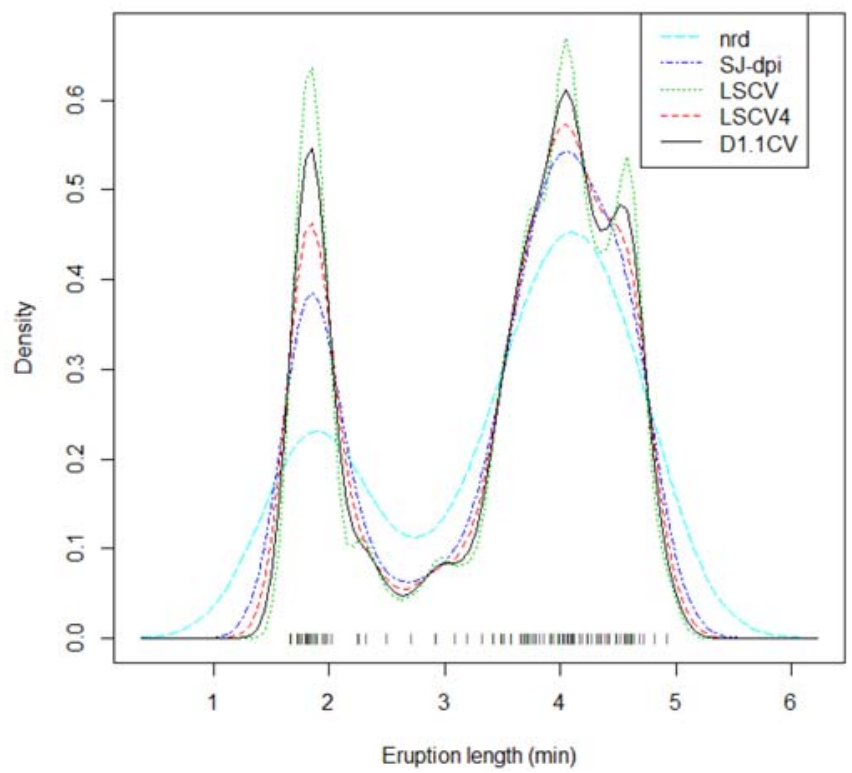

Figure 2. Kernel density estimates with different bandwidths. NR, SJ, $\mathrm{LSCV}, \mathrm{LSCV}_{4}, \mathcal{D}_{1.1} \mathrm{CV}$. 
An important point to note that the density curve for eruption length is similar to bimodal normal density (normal mixture). As commented by Zhang [26], the bandwidth $\widehat{h}_{N R}$ is heavily over smoothes its kernel density curve, underestimating the two peaks of the curve but overestimating the valley between them. On the other hand, $\widehat{h}_{L S C V}$ seems to undersmooth the curve too much, overestimating the two peaks but underestimating for the valley.

However $\widehat{h}_{S J}, \widehat{h}_{L_{S C V}}$, and $\widehat{h}_{\mathcal{D}_{1.1}}$, especially the later, are proper bandwidths for their density estimates to be able to capture the feature of the true density curve.

\section{Conclusion}

The kernel estimator is the most used in density estimation. The main issue is bandwidth selection, which is a hot topic and is still frustrating statisticians. A various bandwidth selection strategies have been proposed such as normal reference $\widehat{h}_{N R}$, unbiased cross-validation $\widehat{h}_{L S C V}$, cross validation, Sheather-Jones method $\widehat{h}_{S J}$ and most recently Zhang [26] proposed a bandwidth $\widehat{h}_{L S C V_{g}}$.

The normal reference bandwidth $\widehat{h}_{N R}$ method is limited the practical use, since they are restricted to situations where a pre-specified family of densities is correctly selected. The popularity of the LSCV method is due to the intuitive motivation and the fact that $\widehat{h}_{L S C V}$ is asymptotically optimal under low conditions. Bandwidth $\widehat{h}_{S J}$ seems to be the best existing bandwidth selector. Unfortunately, it also tends to oversmooth the density estimate when the true density curve is sharp. 
We have attempted to evaluate choice the optimal bandwidth $\widehat{h}_{L S C V}$, using $\beta$-divergence. Compared to traditional bandwidth selection methods designed for kernel density estimation, our proposed $\mathcal{D}_{\beta}$ bandwidth selection method is always one of the best for having large $R E(\widehat{h})$ and small $E\left|\widehat{h} / h_{M I S E}-1\right|$.

Simulation studies showed that our proposed novel optimal bandwidth method designed for kernel density estimation significantly

improves the classical $\widehat{h}_{L S C V}$ for its variability and undersmoothing, adapts to different situations, and out-performs other bandwidths.

\section{References}

[1] A. Basu, I. R. Harris, N. L. Hjort and M. C. Jones, Robust and efficient estimation by minimising a density power divergence, Biometrika 85(3) (1998), 549-559.

DOI: https://doi.org/10.1093/biomet/85.3.549

[2] A. W. Bowman, An alternative method of cross-validation for the smoothing of density estimates, Biometrika 71(2) (1984), 353-360.

DOI: https://doi.org/10.1093/biomet/71.2.353

[3] A. W. Bowman, A comparative study of some kernel-based nonparametric density estimators, Journal of Statistical Computation and Simulation 21(3-4) (1985), 313-327.

DOI: https://doi.org/10.1080/00949658508810822

[4] J. P. Copas and M. J. Fryer, Density estimation and suicide risks in psychiatric treatment, Journal of the Royal Statistical Society: Series A 143(2) (1980), 167-176.

[5] P. Hall, Large sample optimality of least squares cross-validation in density estimation, The Annals of Statistics 11(4) (1983), 1156-1174.

DOI: http://dx.doi.org/10.1214/aos/1176346329

[6] P. Hall, Asymptotic Theory of Minimum Integrated Square Error for Multivariate Density Estimation, In Multivariate Analysis-VI, Editor P. R. Krishnaiah, Elsevier Science, Amsterdam (1985), 289-309.

[7] P. Hall and J. S. Marron, Local minima in cross-validation functions, Journal of the Royal Statistical Society: Series B 53(1) (1991), 245-252. 
[8] W. Hardle, Smoothing Techniques: With Implementation in S, Springer-Verlag, New York, 1991.

[9] M. C. Jones, J. S. Marron and S. J. Sheather, A brief survey of bandwidth selection for density estimation, Journal of the American Statistical Association 91(433) (1996), 401-407.

[10] M. C. Jones and R. F. Kappenman, On a class of kernel density estimate bandwidth selectors, Scandinavian Journal of Statistics 19(4) (1992), 337-349.

[11] Y. Kanazawa, Hellinger distance and Kullback-Leibler loss for the kernel density estimator, Statistics and Probability Letters 18(4) (1993), 315-321.

DOI: https://doi.org/10.1016/0167-7152(93)90022-B

[12] C. R. Loader, Bandwidth selection: Classical or plug-in, The Annals of Statistics 27(2) (1999), 415-438.

DOI: http://dx.doi.org/10.1214/aos/1018031201

[13] J. S. Marron and M. P. Wand, Exact mean integrated squared error, The Annals of Statistics 20(2) (1992), 712-736.

DOI: http://dx.doi.org/10.1214/aos/1176348653

[14] M. Minami and S. Eguchi, Robust blind source separation by beta-divergence, Neural Computation 14(8) (2002), 1859-1886.

DOI: https://doi.org/10.1162/089976602760128045

[15] B. U. Park and B. S. Turlach, Practical performance of several data driven bandwidth selectors, Computational Statistics 7 (1992), 251-270.

[16] E. Parzen, On estimation of a probability density function and mode, Annals of Mathematical Statistics 33(3) (1962), 1065-1076.

[17] M. Rudemo, Empirical choice of histograms and kernel density estimators, Scandinavia Journal of Statistics 9(2) (1982), 65-78.

[18] W. D. Scott, Multivariate Density Estimation: Theory, Practice, and Visualization, Wiley, New York, 1992.

[19] D. W. Scott and G. R. Terrell, Biased and unbiased cross-validation in density estimation, Journal of the American Statistical Association 82(400) (1987), 1131-1146.

[20] S. J. Sheather and M. C. Jones, A reliable data-based bandwidth selection method for kernel density estimation, Journal of the Royal Statistical Society: Series B 53(3) (1991), 683-690.

[21] B. W. Silverman, Density Estimation for Statistics and Data Analysis, Chapman and Hall, London, 1986. 
[22] C. J. Stone, An asymptotically optimal window selection rule for kernel density estimates, The Annals of Statistics 12(4) (1984), 1285-1297.

DOI: http://dx.doi.org/10.1214/aos/1176346792

[23] W. N. Venables and B. D. Ripley, Modern Applied Statistics with S, 4th Edition, Springer, New York, 2002.

[24] M. P. Wand and M. C. Jones, Kernel Smoothing, Chapman and Hall, London, UK, 1995.

[25] S. Weisberg, Applied Linear Regression, Wiley, New York, 1980.

[26] J. Zhang, Generalized least squares cross-validation in kernel density estimation, Statistica Neerlandica 69(3) (2015), 315-328.

DOI: https://doi.org/10.1111/stan.12061 